\title{
A WSN Coalition Formation Algorithm Based on Ant Colony with Dual-Negative Feedback
}

\author{
Na Xia, Jianguo Jiang, Meibin Qi, Chunhua Yu, Yue Huang, and Qi Zhang \\ School of Computer and Information, \\ Hefei University of Technology, \\ Hefei, 230009 PR China \\ jgjiang@hfut.edu.cn
}

\begin{abstract}
In large-scale, complicated Wireless Sensor Networks, the cooperation among sensor nodes is a key topic, and has been receiving more and more attention. The dynamic coalition mechanism in MultiAgent System is an important method for this topic, and then an energyefficient coalition formation algorithm is needed since the energy resource of WSN is restricted. This paper proposes a WSN coalition formation algorithm based on Ant Colony System with dual-negative feedback characteristic. Coalition sponsor inclines to wake those sensor nodes which seldom or never joined coalitions before to form coalition, which can balance the energy consumption among sensor nodes, so as to extend the network lifetime for more tasks. The results of simulation experiment show the validity of this algorithm.
\end{abstract}

Keywords: Wireless Sensor Networks (WSN), Cooperation, Coalition, Ant Colony System.

\section{Introduction}

Wireless sensor networks (WSN) is garnering a lot of research interets due to its important applications such as battlefield surveillance, environment monitoring, home security and target tracking, etc 12 . Due to the severe constraints of energy, computation and communication, sensor nodes have to cooperate with each other to perform tasks. So the cooperation among sensor nodes is a key topic. As the similar characeristics of distribution, autonomy and self-organization between Muti-Agent System (MAS) and WSN, the MAS theory has become an important method for this topic [234]. L. K. Soh and C. Tsatsoulis [567] investigated the WSN cooperation problem adopting dynamic coalition method in MAS. A dynamic sensor node coalition is formed for a task, and the coalition will dismiss after the task is completed. The coalition formation algorithm is based on case-based reasoning (CBR). In the course of coalition formation, the coalition sponsor describes the current task in parameters, searchs in the case database and finds the most similar case according to the task description. Then the corresponding strategy is taken out and modified to suit the current task.

CBR based coalition formation algorithm reduces computation resource spending to a certain extent, but it brings serious disadvantage to the energy 
usage of WSN in other ways: those sensor nodes in successful case would be chosen repeatedly to form coalition to perform tasks, so their limited battery energy will be consumed up too early. The death of these sensor nodes will affect the function of WSN and shorten the network lifetime. This paper proposes a WSN coalition formation algorithm with dual-negative feedback characteristic. It can balance the energy consumption among sensor nodes, so as to extend the every sensor node and network lifetime for more tasks.

\section{The DN-AC Algorithm}

Ant Colony Optimization (ACO) 81910] is inspired by social ants' collective behavior. In Ant Colony System, artificial ants explore solution space, and the transition probability depends on pheromone intensity and heuristic information. The pheromone accumulation (positive feedback) leads ants to consistent good solution, whereas the pheromone evaporation (negative feedback) is helpful to avoid early convergence to suboptimal solution. Based on the feedback mechanism in Ant Colony System, we propose a dual-negative feedback for WSN coalition formation, and design the algorithm for target tracking application. According the movement of target, the algorithm can wake certain sensor nodes dynamically to form coalition to track the target. The tracking accuracy and energy-load balancing are the primary performance metrics for the algorithm.

\subsection{Algorithm}

Define the surveillance zone as $F$, Consider $n$ sensor nodes of WSN randomly scattered in $F$. The sensor node set can be expressed as $A=\left\{A_{1}, \cdots, A_{n}\right\}$, and the position set of sensor nodes can be expressed as $L=\left\{L_{1}, \cdots, L_{n}\right\}$, where $L_{k}$ represents the location of $A_{k}$.

Define the current energy status of $A_{k}$ as pheromone $\tau_{k}, \tau_{k} \in[0,1]$, which can be expressed by the residual energy percentage of $A_{k}$.

When a target $O$ enters $F$, the sensor node $A_{i}$ which first detects the target will become coalition sponsor $A_{\text {sponsor }}$. It will wake certain sensor nodes to form coalition for tracking mainly considering their pheromone value (energy status) and the distance from these sensor nodes to the target. The waking probability of $A_{k}$ at time $t$ is calculated as follows:

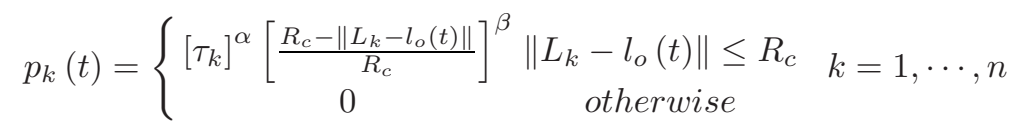

Here, $l_{o}(t)$ is the located position of target $O$ at time $t . R_{c}$ is the detection radius of sensor node. It is evident that larger $\tau_{k}$ and smaller distance means that the sensor node has more chance to be wakened.

Wake sensor nodes stochastically according to $p_{k}(t)$. The wakened sensor nodes form coalition to track the target for $\Delta t$ period, and then update the waking probability $p_{k}(t)$ using the newly located position of target $O$, so as to 
execute the next waking operation. With the target moving, the sensor node coalition is dynamic in the surveillance zone. While target moves out of $F$, a round of target tracking is over.

The wakened/sleeping state of sensor node $A_{k}$ at time $t$ can be represented by the indicator $\theta_{k}(t)$ as follows:

$$
\theta_{k}(t)=\left\{\begin{array}{l}
1 A_{k} \text { is wakened at time } t \\
0 A_{k} \text { is sleeping at time }
\end{array} \quad k=1, \cdots, n\right.
$$

The integral working time of sensor node $A_{k}$ in dynamic coalition during a round of target tracking can be represented as follows:

$$
T_{k}=\sum_{t} \theta_{k}(t) k=1, \cdots, n
$$

After a round of target tracking, the pheromone value on every sensor node will be updated. For those sensors which participated in dynamic coalition, besides pheromone evaporation, their pheromone will be decreased further, which is we termed Dual-Negative feedback. The updated pheromone value for $A_{k}$ is calculated as follows:

$$
\tau_{k} \leftarrow \rho \cdot \tau_{k}-\Delta \tau_{k} k=1, \cdots, n
$$

Here $1-\rho \in(0,1)$ is the decay coefficient which represents the energy consumption under sleeping model. The pheromone decrease $\Delta \tau_{k}$ represents the energy consumption of sensor node $A_{k}$ during this round of target tracking, which is as follows:

$$
\Delta \tau_{k}=\left\{\begin{array}{c}
\xi \cdot \frac{T_{k}}{\sum_{k} T_{k}} \text { if } A_{k} \text { participate in dynamic coalition } \\
0 \text { otherwise }
\end{array} \quad k=1, \cdots, n\right.
$$

$\Delta \tau_{k}$ is direct proportion to $T_{k}$. Define the pheromone decrease coefficient as $\xi$, which can be adjusted.

Consequently, when next target appears, it is less possible for $A_{\text {sponsor }}$ to wake the sensor nodes which participated in coalition before, whereas $A_{\text {sponsor }}$ inclines to wake those sensor nodes which seldom or never joined coalition to form coalition. This behavior balances energy-load among sensor nodes, and network lifetime extending is achieved.

To guarantee the tracking reliability and avoid waste of resource, we define the minimal node number $w_{\min }\left(w_{\min } \geq 3\right)$ and maximal node number $w_{\max }$ for a dynamic coalition. After $N C_{\max }$ rounds of target tracking, query network, and update $\tau_{k}(t)$ using the actual energy status of each sensor. This operation is good to the correctness of latter decision for algorithm.

We term this dynamic coalition formation algorithm based on Ant Colony with Dual-Negative feedback as DN-AC, and its pseudocode is as follows:

- Step1. Initialize: Set initial value $\tau_{k}=1$ (100\%).

- Step2. Set $N C=0$. 
- Step3. Set $t=0, \theta_{k}(t)=0, T_{k}=0, \Delta \tau_{k}=0$.

- Step4. If ( $O$ is detected by $A_{i}$ ) then $\left\{A_{i}\right.$ becomes $A_{\text {sponsor }}, l_{o}(t)=L_{i}$;

$A_{\text {sponsor }}$ wakes certain sensor nodes to form coalition according to $p_{k}(t)$ by (1), update $\theta_{k}(t)$, the coalition tracking $O$ for $\Delta t$ period, Set $t=t+\Delta t$, update $\left.l_{o}(t)\right\}$

Else wait.

- Step5. While $\left(l_{o}(t) \in F\right)$ $\left\{A_{\text {sponsor }}\right.$ wakes certain sensor nodes to form coalition according to $p_{k}(t)$ by $(1)$, and selects the sensor node with maximal $p_{k}(t)$ value as new $A_{\text {sponsor }}$, update $\theta_{k}(t)$, the coalition tracking $O$ for $\Delta t$ period, $t=t+\Delta t$, update $\left.l_{o}(t)\right\}$.

- Step6. Output $l_{o}(t), t=0, \Delta t, 2 \Delta t, 3 \Delta t, \cdots$.

- Step7. For $k=1$ to $n$ do Update the pheromone $\tau_{k}$ by $(3),(4),(5)$.

- Step8. Set $N C=N C+1$.

- Step9. If $\left(N C<N C_{\max }\right)$ then Goto step 3

Else query network, update the $\tau_{k}$ using the actual energy status of each sensor node, Goto step 2.

\subsection{Algorithm Evaluation}

The performance metrics being investigated are:

(1) Tracking error of target $O$

Define the tracking error of target $O$ as follows:

$$
\varphi(O)=\overline{\left|l_{o}(t)-l_{o}^{*}(t)\right|}
$$

Where $l_{o}(t)$ is the target position located by the sensor node coalition at time $t$, while $l_{o}^{*}(t)$ is the actual position of target at time $t$. So, $\varphi(O)$ represents the accuracy of target tracking.

(2) Mean square error of network energy

Define the mean square error of network energy at time $t$ as follows:

$$
\sigma(t)=\sqrt{\frac{1}{n} \sum_{i=1}^{n}\left(\tau_{i}-\bar{\tau}\right)^{2}}
$$

Here $\tau_{1}, \cdots, \tau_{n}$ are the pheromone values of $n$ sensor nodes at time $t$. This metric represents the status of network energy. The smaller the value is the more energy load-balanced the network achieves.

(3) Health degree of network

Define the total number of living sensor nodes (i.e. not run out of energy) at time $t$ as $n_{\text {living }}(t)$. The health degree of network at time $t$ can be defined:

$$
H(t)=\frac{n_{\text {living }}(t)}{n}
$$


This metric represents the sensor protection performance of the algorithm. Otherwise, the network lifetime $T$ can be defined as the time period from the instant the network is deployed to the moment when $30 \%$ of all sensor nodes run out of energy.

\section{Simulation Experiment}

Fig.1 depicts the surveillance zone, which is a rectangle from $(0 \mathrm{~m}, 0 \mathrm{~m})$ to $(200 \mathrm{~m}, 200 \mathrm{~m})$. There are 500 sensor nodes shown by dots randomly scattered in the zone. In turn, the targets $O_{1}, O_{2}, O_{3}, O_{4}, O_{5}$ and $O_{6}$ move from the same start point $(0,0)$ with constant velocity but different angles $35^{\circ}, 38.7^{\circ}, 42^{\circ}, 45^{\circ}, 47.7^{\circ}$ and $50.2^{\circ}$ to cross the surveillance zone within $40 \mathrm{~s}$. These targets' trajectory is represented by dashed line.

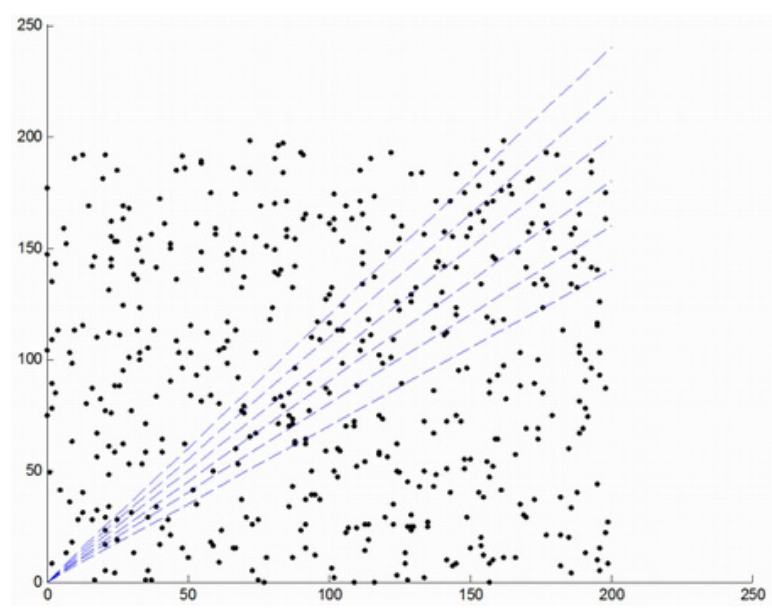

Fig. 1. Surveillance zone

We adopt CBR method and DN-AC algorithm to form dynamic coalition for target tracking respectively, and compare 50 times Monte Carlo simulation results. The parameters of DN-AC are shown in Table 1.

For DN-AC, it forms dynamic coalition to track $O_{1}$, and the tracking result is depicted in Fig.2. The red circles represent the sensor nodes wakened

Table 1. Parameters setting

\begin{tabular}{ccccccccc}
\hline$R_{c}$ & $\Delta t$ & $\tau_{k}$ & $\alpha$ & $\beta$ & $\rho$ & $\xi$ & $w_{\min }$ & $w_{\max }$ \\
\hline $40 m$ & $2 s$ & 1 & 1 & 1 & 0.99 & 20 & 3 & 5 \\
\hline
\end{tabular}


to form dynamic coalition during this round of target tracking, moreover the continuous green line represents the trail gained by these sensor nodes locating $O_{1}$. The tracking error of this round is $1.19 \mathrm{~m}$. In Fig.3, the tracking errors of $O_{1}, O_{2}, O_{3}, O_{4}, O_{5}$ and $O_{6}$ for $\mathrm{CBR}$ and $\mathrm{DN}$-AC are compared. The result shows the tracking accuracy of the two algorithms is similar.

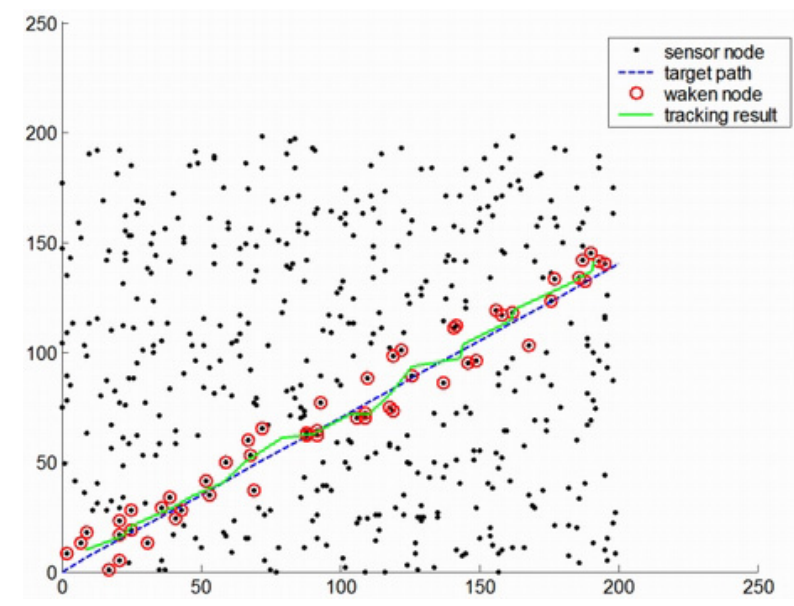

Fig. 2. Tracking result to $O_{1}$ using DN-AC, $\varphi\left(O_{1}\right)=1.19 m$

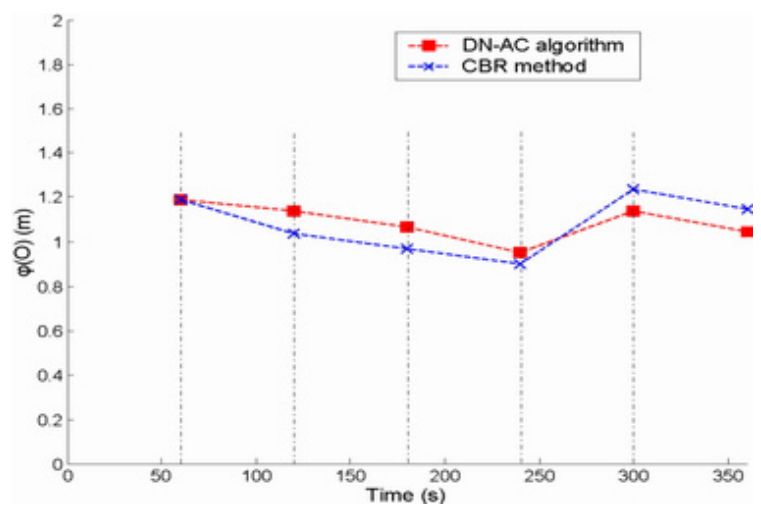

Fig. 3. The comparison of tracking errors of 6 targets between two algorithms

During the 6 rounds of target tracking, the variance of the mean square error of network energy $\sigma(t)$ and the health degree of network $H(t)$ for two algorithms are depicted in Fig.4 and 5. In Fig.4, for CBR, the $\sigma(t)$ value rises rapidly because CBR method results in some sensor nodes running out of energy too early and some other nodes consuming energy badly, so that the energy level of 
network is rather separate. Since DN-AC can balance the energy consumption among sensor nodes, the $\sigma(t)$ value rises more slowly than CBR. In Fig.5, It is evident that DN-AC exhibits better network health degree than CBR. It is found that there are 30 sensor nodes dead after the 6 th round of target tracking for CBR (shown in Fig.6), whereas the number is 15 for DN-AC (shown in Fig.7). Therefore DN-AC achieves significantly better energy-load balancing performance than CBR, so as to extend the network lifetime effectively.

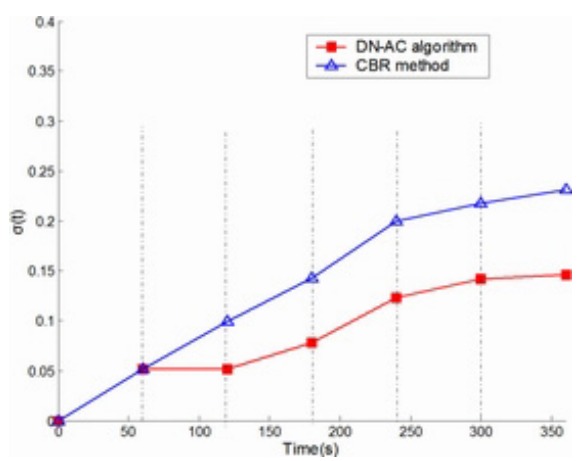

Fig. 4. The variance of mean square error of network energy for two algorithms

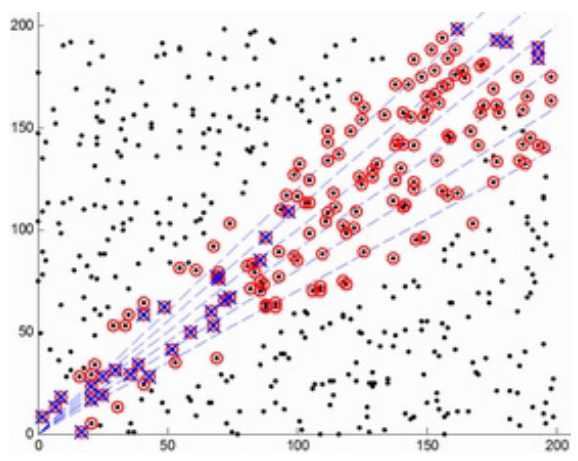

Fig. 6. The status of dead sensor nodes after the 6th round of target tracking for CBR, the crosses representing the dead sensor nodes

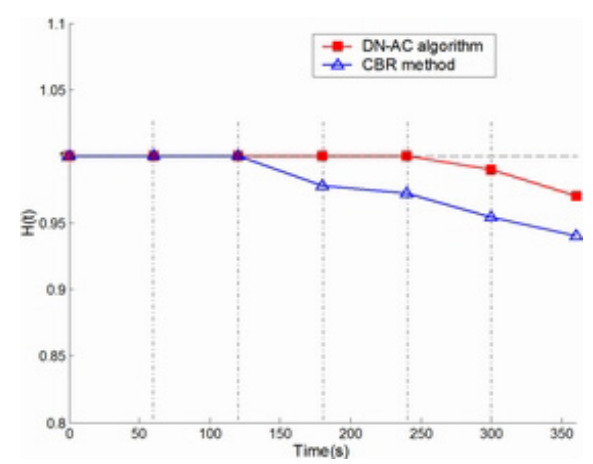

Fig. 5. The variance of health degree of network for two algorithms

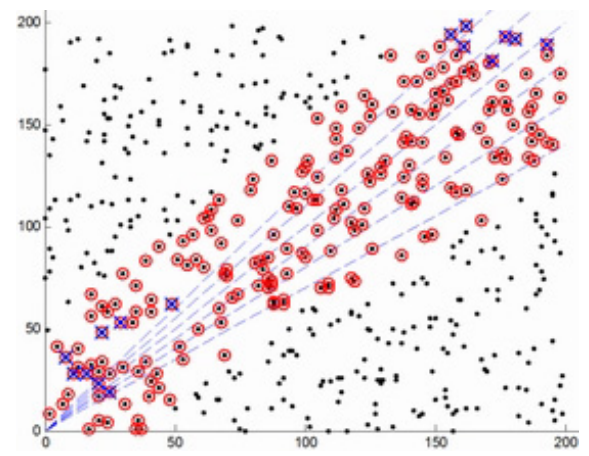

Fig. 7. The status of dead sensor nodes after the 6 th round of target tracking for $\mathrm{DN}-\mathrm{AC}$, the crosses representing the dead sensor nodes

\section{Conclusions}

This paper investigates the energy-efficient dynamic coalition formation algorithm in WSN, and proposes a coalition formation algorithm based on Ant 
Colony System with dual-negative feedback characteristic. With coalition quality guaranteed, it can balance the energy consumption among sensor nodes, so as to extend the network lifetime for more tasks. The results of simulation experiment show the validity of this algorithm for target tracking application.

Acknowledgments. This work is supported by NSFC, National Natural Science Foundation of China, No. 60474035, and Natural Science Foundation of Anhui Province, No.070412035.

\section{References}

1. L. F. Akyildiz, W. Su, Y. Survey on Sensor Networks. IEEE Communication Magazine, 2002, 40(8): 102-114.

2. H. B. Yu, P. Zeng, W. Liang. Intelligent Wireless Sensor Networks. Beijing: Science Press, 2006. 215-278.

3. C. Che, W. Liang, Y. Zhou, et al. Cooperation Problem of Wireless Sensor Network Based on Multi-agent. Chinese Journal of Scientific Instrument, 2005, 26(8A): 229-232.

4. V. Lesser, C. L. Ortiz, M. Tambe. Distributed Sensor Networks: A Multiagent Perspective. Kluwer Academic Publishers, 2003.

5. L. K. Soh, C. Tsatsoulis. Real-time Satisfying Multiagent Coalition Formation. In Working Notes of AAAI Workshop on Coalition Formation in Dynamic Multiagent Environment. 2002. 7-15.

6. L. K. Soh, X. Li. An Integrated Multi-Level Learning Approach to Multiagent Coalition Formation. In Proceedings of IJCAI'03. Acapulco, Mexico, 2003. 619-624.

7. L. K. Soh, C. Tsatsoulis. Satisfying Coalition Formation among Agents. In Proceedings of AAMAAS'02. Bologna, Italy, 2001. 15-19.

8. M. Dorigo, T. Stutzle. Ant colony optimization. MIT Press, U. S., 2004.

9. R. Wang, Y. Liang, G. Q. Ye, et al. Swarm Intelligence for the Self-Organization of Wireless Sensor Network. In Proceedings of the IEEE Congress on Evolutionary Computation. Vancouver, BC, Canada, 2006. 838-842.

10. S. Selvakennedy, S. Sinnappan and Y. Shang. T-ANT: A Nature-Inspired Data Gathering Protocol for Wireless Sensor Networks. Journal of Communications, 2006, 1(2): 22-29. 